\title{
Laboratory and radio-astronomical spectroscopy of the hyperfine structure of $\mathrm{N}_{2} \mathrm{D}^{+\star}$
}

\author{
L. Dore ${ }^{1}$, P. Caselli ${ }^{2}$, S. Beninati ${ }^{1}$, T. Bourke ${ }^{3}$, P. C. Myers ${ }^{3}$, and G. Cazzoli ${ }^{1}$ \\ 1 Dipartimento di Chimica “G. Ciamician”, Università di Bologna, via Selmi 2, 40126 Bologna, Italy \\ 2 INAF - Osservatorio Astrofisico di Arcetri, Largo E. Fermi 5, 50125 Firenze, Italy \\ e-mail: caselli@arcetri.astro.it \\ ${ }^{3}$ Harvard-Smithsonian Center for Astrophysics, 60 Garden Street, Cambridge, MA 02138, USA
}

Received 30 June 2003 / Accepted 30 September 2003

\begin{abstract}
We present the first laboratory measurements of the hyperfine structure of the $J=1 \leftarrow 0$ rotational transition of $\mathrm{N}_{2} \mathrm{D}^{+}$, a good tracer of the dense regions of molecular cloud cores, and the spectra of unresolved high $J$ transitions recorded in the 308-463 GHz region. Together with a high sensitivity radio-astronomical spectrum of the $\mathrm{N}_{2} \mathrm{D}^{+} J=1 \rightarrow 0$ rotational transition in a quiescent cloud core, we determined with high precision the frequencies of the seven hyperfine components and the molecular spectroscopic constants, allowing us to make predictions on the $\mathrm{N}_{2} \mathrm{D}^{+}$frequencies of higher $J$ transitions occurring in the submillimeter-wave region.
\end{abstract}

Key words. molecular data - methods: laboratory - ISM: individual: L183 - molecules - radio lines: ISM

\section{Introduction}

Deuterium fractionation is an active process in cold and dense molecular clouds, mainly due to the deuteron-proton exchange reaction $\mathrm{H}_{3}^{+}+\mathrm{HD} \rightleftharpoons \mathrm{H}_{2} \mathrm{D}^{+}+\mathrm{H}_{2}$, which is exothermic by $\sim 220 \mathrm{~K}$ (Millar et al. 1989) in the forward direction. Once formed, $\mathrm{H}_{2} \mathrm{D}^{+}$transfers the deuteron to abundant gaseous neutral species, such as $\mathrm{CO}$ and $\mathrm{N}_{2}$, producing $\mathrm{DCO}^{+}$and $\mathrm{N}_{2} \mathrm{D}^{+}$. Indeed, the $N\left(\mathrm{DCO}^{+}\right) / N\left(\mathrm{HCO}^{+}\right)$and $N\left(\mathrm{~N}_{2} \mathrm{D}^{+}\right) / N\left(\mathrm{~N}_{2} \mathrm{H}^{+}\right)$column density ratios observed towards cold cloud cores (e.g. Butner et al. 1995; Caselli et al. 2002b) are much larger ( $>1000$ times) than the elemental abundance of deuterium ([D]/[H] 1.6 $\times 10^{-5}$; Linsky et al. 1995), making $\mathrm{DCO}^{+}$and $\mathrm{N}_{2} \mathrm{D}^{+}$two good tracers of physical and chemical conditions of molecular cloud cores, and allowing the determination of the electron fraction (e.g. Guélin et al. 1982; Caselli et al. 1998; Williams et al. 1998; Caselli et al. 2002b), fundamental parameter which controls the dynamical evolution of molecular clouds and regulates the star formation rate (McKee 1989).

At $\mathrm{H}_{2}$ number densities larger than about $10^{5} \mathrm{~cm}^{-3}$ (and dust temperatures $\$ 20 \mathrm{~K}$ ), another process becomes important for cloud core evolution: the differential molecular freeze out onto dust grains. Evidences of gas phase $\mathrm{CO}$ depletions have been found toward starless cores (Willacy et al. 1998; Caselli et al. 1999; Bacmann et al. 2002; Bergin et al. 2002;

Send offprint requests to: L. Dore, e-mail: dore@ciam. unibo.it

* Tables 4 to 8 are only available in electronic form at the CDS via anonymous ftp to cdsarc.u-strasbg.fr $(130.79 .128 .5)$ or via http://cdsweb.u-strasbg.fr/cgi-bin/qcat?J/A+A/413/1177
Tafalla et al. 2002) and star forming regions (McMullin et al. 1994; Kramer et al. 1999; Jorgensen et al. 2002). In particular, the central zones of starless cores, future sites of star formation, are likely to be almost completely deprived of gaseous $\mathrm{CO}$ molecules, so that the abundance of related species such as $\mathrm{HCO}^{+}$and $\mathrm{DCO}^{+}$is also drastically reduced (e.g. Caselli et al. 2002b; Lee et al. 2003). On the other hand, $\mathrm{N}_{2} \mathrm{H}^{+}$and $\mathrm{N}_{2} \mathrm{D}^{+}$are not significantly affected by depletion (Caselli et al. 1999; Bergin et al. 2001; Tafalla et al. 2002), given that the parent species $\mathrm{N}_{2}$ is more volatile than $\mathrm{CO}$ and thus it maintains a large fractional abundance even at densities as large as $10^{6} \mathrm{~cm}^{-3}$ (Bergin \& Langer 1997; Aikawa et al. 2001; Caselli et al. 2002b; Aikawa et al. 2003). Moreover, deuterium fractionation increases with depletion of neutral species reacting with $\mathrm{H}_{3}^{+}$and $\mathrm{H}_{2} \mathrm{D}^{+}$, such as CO (e.g. Dalgarno \& Lepp 1984; Roberts \& Millar 2000; Bacmann et al. 2003). Thus, the $\mathrm{N}_{2} \mathrm{D}^{+} / \mathrm{N}_{2} \mathrm{H}^{+}$abundance ratio and the $\mathrm{N}_{2} \mathrm{D}^{+}$fractional abundance increase towards the core center, so that $\mathrm{N}_{2} \mathrm{D}^{+}$is particularly suitable to investigate the kinematics as well as the physical and chemical conditions of the highly $\mathrm{CO}-$ depleted core nuclei. In fact, $\mathrm{N}_{2} \mathrm{D}^{+}$observations have recently allowed an accurate analysis of the velocity field and kinematics across the core nucleus of L1544, a well-known starless core (Caselli et al. 2002a). $\mathrm{N}_{2} \mathrm{D}^{+}$lines can be used to determine the line of sight component of the velocity of ions in the densest portions of cloud cores. These values can then be compared with those obtained from lines of chemically related neutral species (e.g. $\mathrm{NH}_{2} \mathrm{D}$, observable with similar angular resolution), to estimate the speed of the ion-neutral drift 
in the core nucleus and to quantify the importance of magnetic fields in the dynamical evolution of star forming clouds.

In summary, it is extremely important to determine with high precision the frequencies of $\mathrm{N}_{2} \mathrm{D}^{+}$rotational lines for kinematic studies (see e.g. Lee et al. 2001). Also, a more precise determination of the $\mathrm{N}_{2} \mathrm{D}^{+}$spectroscopic constants will be important to more accurately derive $\mathrm{N}_{2} \mathrm{D}^{+}$column densities and abundances, given that the hyperfine splitting allows a direct determination of the transition optical depth.

The dominant hyperfine interactions in $\mathrm{N}_{2} \mathrm{D}^{+}$are those between the molecular electric field gradient and the electric quadrupole moments of the two nitrogen nuclei: for the $J=1 \rightarrow 0$ rotational transition of $\mathrm{N}_{2} \mathrm{H}^{+}$, these produce a splitting into seven hyperfine components (Caselli et al. 1995). The quadrupole coupling of the D nucleus, with spin 1, may add complexity to the hyperfine structure of $\mathrm{N}_{2} \mathrm{D}^{+}(1-0)$, but its effect is likely to be negligible (see Sect. 3).

The hyperfine structure of the $J=1 \leftarrow 0$ transition of $\mathrm{N}_{2} \mathrm{D}^{+}$was observed for the first time by Anderson et al. (1977), who were able to detect the $\Delta F=0$ and +1 components due to the outer $\mathrm{N}$ nucleus; all the three components were later detected in L134 by Snyder et al. (1977). Recently, Turner (2001) has reported observations of $\mathrm{N}_{2} \mathrm{D}^{+}(1-0)$ in several astronomical objects where the hyperfine structure due to both the $\mathrm{N}$ nuclei is fully resolved into seven components.

As far as higher $J$ transitions are concerned, Sastry et al. (1981) observed unresolved rotational lines up to the $J=$ $6 \leftarrow 5$ one in the positive column of a DC glow discharge, while some hyperfine structure for the $J=2 \rightarrow 1$ and $3 \rightarrow 2$ transitions has been detected by several radioastronomers (Gerin et al. 2001; Turner 2001; Caselli et al. 2002a).

In this paper, we report the first laboratory measurements of the $\mathrm{N}_{2} \mathrm{D}^{+}(1-0)$ hyperfine structure and we compare these measurements with a high sensitivity astronomical observation of the same transition toward the dense cloud core L183. This analysis allowed us to derive all the determinable hyperfine parameters. In addition, laboratory spectra of unresolved high $J$ transitions were recorded up to $463 \mathrm{GHz}$, in order to determine accurate rotational and distortion constants for predictions in the submillimeter-wave region.

\section{Observations}

\subsection{Laboratory measurements}

The laboratory spectrum was observed with a frequencymodulated millimeter-wave spectrometer (Cazzoli \& Dore 1990a) equipped with a double-pass negative glow discharge cell (Dore et al. 1999). The radiation source was a frequency multiplier driven by a Gunn oscillator working in the region 77-116 GHz (J. E. Carlstrom Co) or the Gunn itself for measurements at $77 \mathrm{GHz}$; the signal, detected by a liquidhelium-cooled InSb hot electron bolometer (QMC Instr. Ltd. type QFI/2), was demodulated at 2- $f$ by a lock-in amplifier.

$\mathrm{N}_{2} \mathrm{D}^{+}$was produced by discharging a $1: 1$ mixture of $\mathrm{N}_{2}$ and $\mathrm{D}_{2}$ in Ar buffer gas with a total pressure of about 5 mTorr. The discharge current was a few $\mathrm{mA}$, the cell was cooled at $77 \mathrm{~K}$ by liquid nitrogen circulation, and an axial magnetic
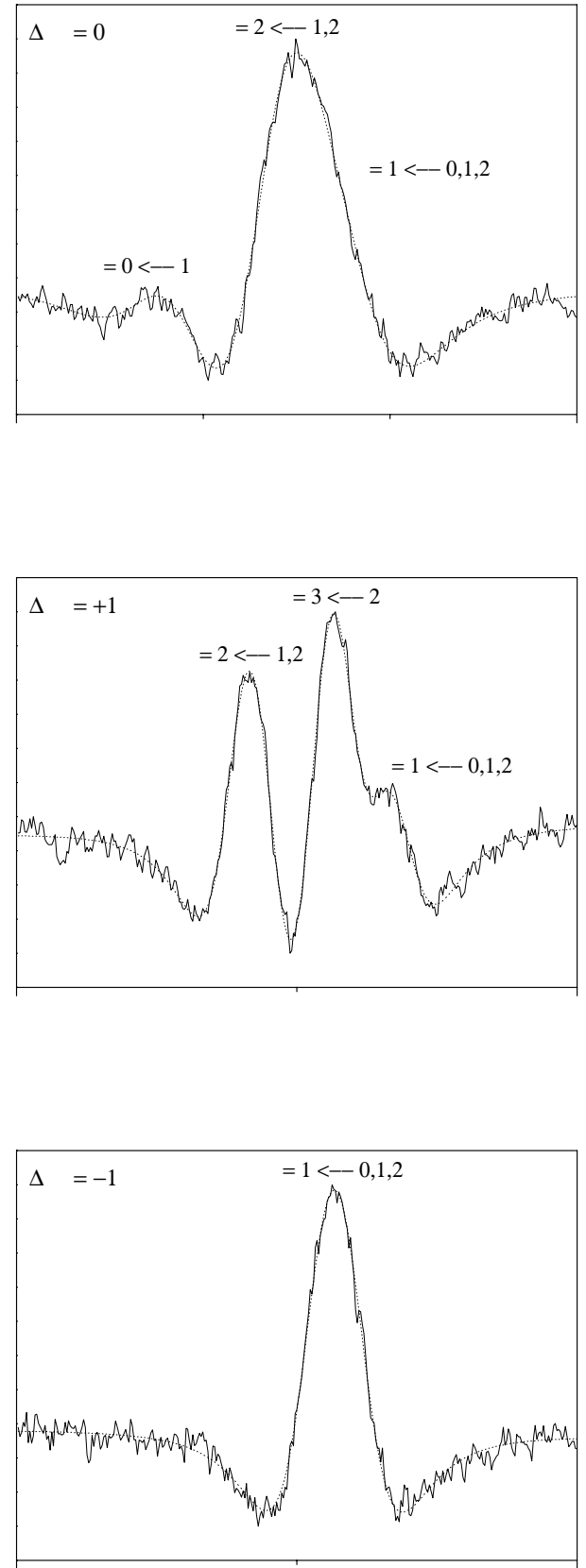

Fig. 1. The three hyperfine components of the $J=1 \leftarrow 0$ transition of $\mathrm{N}_{2} \mathrm{D}^{+}$due to the outer $\mathrm{N}$ nucleus. The $\Delta F_{1}=0$ and +1 components show the hyperfine structure due to the inner $\mathrm{N}$ nucleus: their profile (continuous) has been fit to a model lineshape (dotted) to recover the hyperfine frequencies.

field up to $165 \mathrm{G}$ was applied throughout the length of the discharge. With this longitudinal magnetic field applied, ions are produced and observed in the negative glow (De Lucia et al. 1982), which is a nearly field free region and where they are expected to show negligible Doppler shift due to the drift velocity, occurring, instead, in the positive column (Sastry et al. 1981) where a low axial electric field is present. In addition, the double-pass arrangement would compensate for such a shift, if present. Figure 1 shows the hyperfine structure of the $J=1 \leftarrow 0$ transition of $\mathrm{N}_{2} \mathrm{D}^{+}$observed in laboratory. 


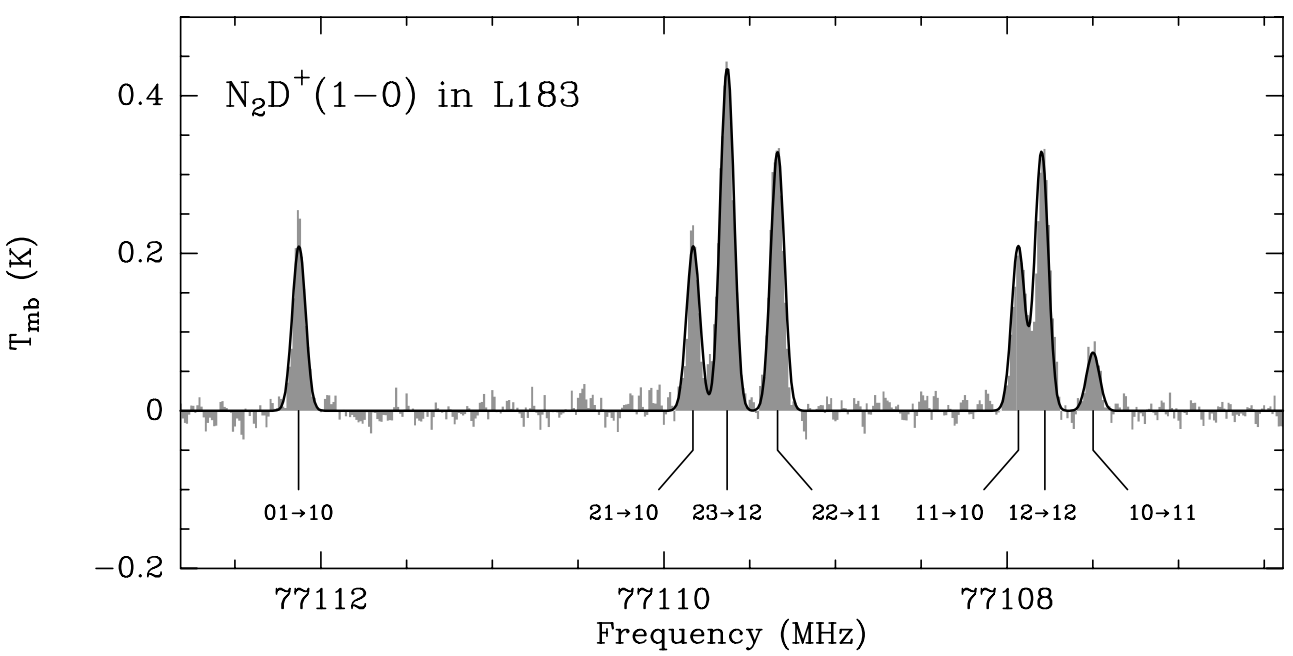

Fig. 2. Spectrum of the $J=1 \rightarrow 0$ transition of $\mathrm{N}_{2} \mathrm{D}^{+}$towards the starless core L183 (grey histogram). The black curve is the hfs fit obtained with the data reduction package CLASS (see URL www.iram.fr/IRAMFR/GS/class/class.html) using the frequencies of the seven hyperfine components as found in this paper. The fit parameters are: excitation temperature $T_{\mathrm{ex}}=4.1 \pm 0.5 \mathrm{~K}$, LSR velocity $V_{\mathrm{LSR}}=2.444 \pm 0.001 \mathrm{~km} \mathrm{~s}^{-1}$, line width $\Delta v=0.331 \pm 0.004 \mathrm{~km} \mathrm{~s}^{-1}$, and total optical depth $\tau_{\text {TOT }}=1.8 \pm 0.3$. Each hyperfine is indicated by the corresponding $F_{1}, F$ quantum numbers (see also Table 1).

\subsection{Detection toward L183}

The observations of L183 were performed with the NRAO $12-\mathrm{m}$ telescope ${ }^{1}$ (now the Steward Observatory 12-m telescope) during October $1999\left(\mathrm{~N}_{2} \mathrm{H}^{+}\right)$and February 2000 $\left(\mathrm{N}_{2} \mathrm{D}^{+}\right)$. The position observed was the peak of the $\mathrm{N}_{2} \mathrm{H}^{+}(1-0)$ integrated emission map made by Lee et al. (2001) $\left(\mathrm{RA}(\mathrm{J} 2000)=15^{\mathrm{h}} 54^{\mathrm{m}} 06^{\mathrm{s}} \cdot 5, \operatorname{Dec}(\mathrm{J} 2000)=-02^{\circ} 52^{\prime} 23^{\prime \prime}\right)$. Two orthogonal polarizations were observed simultaneously and averaged together to produce the final spectra. All observations were performed in frequency switching mode, with a throw of $\pm 9 \mathrm{MHz}$. The backend used was the Millimeter Autocorrelator, configured with a channel spacing of $6.1 \mathrm{kHz}$, corresponding to a spectral resolution of $12.2 \mathrm{kHz}$. This coverts to a velocity resolution of $0.039 \mathrm{~km} \mathrm{~s}^{-1}$ for $\mathrm{N}_{2} \mathrm{H}^{+}(1-0)(93.2 \mathrm{GHz})$ and $0.047 \mathrm{~km} \mathrm{~s}^{-1}$ for $\mathrm{N}_{2} \mathrm{D}^{+}(1-0)(77.1 \mathrm{GHz})$. The respective beam sizes $(F W H M)$ are $68^{\prime \prime}$ at $93.2 \mathrm{GHz}$ and $82^{\prime \prime}$ at $77.1 \mathrm{GHz}$.

System temperatures were typically $200 \mathrm{~K}$ during the observations. The spectra have been corrected for the main beam efficiency and are in unit of $T_{\mathrm{mb}}$. The final spectra have rms per channel sensivities of $14 \mathrm{mK}\left(\mathrm{N}_{2} \mathrm{H}^{+}(1-0)\right)$ and $15 \mathrm{mK}$ $\left(\mathrm{N}_{2} \mathrm{D}^{+}(1-0)\right)$. With a peak of $T_{\mathrm{mb}} \approx 1.6 \mathrm{~K}$, the $\mathrm{N}_{2} \mathrm{H}^{+} 1-0$ spectrum has a peak signal-to-noise ratio of over 100 . The $\mathrm{N}_{2} \mathrm{D}^{+}(1-0)$ spectrum is shown in Fig. 2. It is interesting to note that this transition does not present excitation anomalies, unlike $\mathrm{N}_{2} \mathrm{H}^{+}(1-0)$ (see Caselli et al. 1995).

\section{Analysis and discussion}

The frequencies of the seven hyperfine components of $\mathrm{N}_{2} \mathrm{D}^{+}(1-0)$ observed toward the dense cloud L183 (see Fig. 2) have been determined by comparing their velocities with those obtained from the $\mathrm{N}_{2} \mathrm{H}^{+}(1-0)$ spectrum, and fixing the

1 The National Radio Astronomy Observatory is a facility of the National Science Foundation operated under cooperative agreement by Associated Universities, Inc.
$\mathrm{N}_{2} \mathrm{H}^{+}(1-0)$ frequency to $93176.2608 \mathrm{MHz}$ for the $F_{1} F=$ $01 \rightarrow 12$ component. This value has been obtained from Lee et al. (2001), where a comparison between the velocities of the $F_{1} F=01 \rightarrow 12$ component of the $\mathrm{N}_{2} \mathrm{H}^{+}(1-0)$ line with that of $\mathrm{C}^{18} \mathrm{O}(1-0)$ was considered, but using the new $\mathrm{C}^{18} \mathrm{O}(1-0)$ frequency, recently redetermined with high precision $(0.15 \mathrm{kHz}$, see Cazzoli et al. 2003). Caselli et al. (1995) deduced a value which is $4.2 \mathrm{kHz}$ more than that one deduced here; on the other hand, they gauged the $\mathrm{N}_{2} \mathrm{H}^{+}(1-0)$ frequency from the comparison with the $\mathrm{C}_{3} \mathrm{H}_{2}\left(2_{1,2} \rightarrow 1_{0,1}\right)$ line, measured in the laboratory with an uncertainty of $6 \mathrm{kHz}$ (Vrtilek et al. 1987). However, although the $\mathrm{C}^{18} \mathrm{O}(1-0)$ frequency is known with higher precision than the $\mathrm{C}_{3} \mathrm{H}_{2}$ line, we note that $\mathrm{N}_{2} \mathrm{H}^{+}$and $\mathrm{CO}$ do not trace the same regions in low mass cloud cores (the latter being significantly depleted in the central regions, where the former peaks; see e.g. Tafalla et al. 2002), whereas $\mathrm{C}_{3} \mathrm{H}_{2}$ and $\mathrm{N}_{2} \mathrm{H}^{+}$seem to have a similar behaviour (Benson et al. 1998). CO freeze out is also present in L1512 (see Lee et al. 2003), the quiescent core where all the above frequency determinations have been performed. Therefore, the presence of velocity fields along the line of sight of L1512 may cause a shift between $\mathrm{N}_{2} \mathrm{H}^{+}$and $\mathrm{C}^{18} \mathrm{O}$ lines, although current kinematic studies present L1512 as a dynamically stable core (Lee et al. 2003).

For the above reason, it is thus very important to determine a precise value of the $\mathrm{N}_{2} \mathrm{H}^{+}(1-0)$ frequency in the laboratory, to overcome the radioastronomical uncertainties. However, this is a difficult task, since sub-Doppler spectroscopy can not be exploited with electric discharges, and pressure line shift may occur (see Buffa et al. 1994): we are actually working on this (Dore et al., in preparation).

The measured hyperfine frequencies, both from laboratory and space, are listed in Table 1, and the determined spectroscopic constants are reported in Table 2, where the hyperfine constants of $\mathrm{N}_{2} \mathrm{H}^{+}$are also reported for comparison purposes. The error associated with the radioastronomical determination of $v_{0}$ does not include the $6 \mathrm{kHz}$ uncertainty in the $\mathrm{N}_{2} \mathrm{H}^{+}(1-0)$ 
Table 1. Hyperfine transition frequencies (MHz) and residuals $(\mathrm{MHz})$ of $\mathrm{N}_{2} \mathrm{D}^{+}$.

\begin{tabular}{|c|c|c|c|c|c|c|c|c|c|c|}
\hline \multirow[b]{2}{*}{$J^{\prime}$} & \multirow[b]{2}{*}{$F_{1}^{\prime}$} & \multirow[b]{2}{*}{$F^{\prime}$} & \multirow[b]{2}{*}{$J$} & \multirow[b]{2}{*}{$F_{1}$} & \multirow[b]{2}{*}{$F^{a}$} & \multicolumn{2}{|c|}{ Laboratory } & \multicolumn{2}{|c|}{ Radioastronomy } & \multirow[b]{2}{*}{ Lab.-radio. } \\
\hline & & & & & & Obs. $^{b}$ & Obs.-calc. & Obs. $^{c}$ & Obs.-calc. & \\
\hline 1 & 1 & 0 & 0 & 1 & 1 & 77107.498 & -0.002 & 77 107.4872(23) & ) 0.0020 & 0.011 \\
\hline 1 & 1 & 2 & 0 & 1 & 1,2 & 77107.798 & 0.001 & 77 107.7753(8) & -0.0012 & 0.023 \\
\hline 1 & 1 & 1 & 0 & 1 & $0,1,2$ & 77107.934 & 0.001 & 77 107.9147(13) & ) 0.0028 & 0.019 \\
\hline 1 & 2 & 2 & 0 & 1 & 1,2 & 77109.338 & 0.002 & 77 109.3342(5) & 0.0000 & 0.004 \\
\hline 1 & 2 & 3 & 0 & 1 & 2 & 77109.632 & -0.002 & 77 109.6259(5) & 0.0001 & 0.006 \\
\hline 1 & 2 & 1 & 0 & 1 & $0,1,2$ & 77109.830 & 0.000 & 77 109.8196(8) & -0.0003 & 0.010 \\
\hline 1 & 0 & 1 & 0 & 1 & $0,1,2$ & 77112.130 & 0.000 & 77 112.1180(8) & 0.0000 & 0.012 \\
\hline
\end{tabular}

${ }^{a}$ The rotational ground level is split in three states with $F=F_{1}+I_{N}=0,1,2$ : they are degenerate because $J=0$.

${ }^{b}$ Statistical uncertainty estimated from repeated measurements is about $5 \mathrm{kHz}$ for well resolved components; however, line frequencies may be shifted by effect of the pressure (see Buffa et al. 1994) of about $+10-15 \mathrm{kHz}$.

${ }^{c}$ Quoted errors do not take into account of the uncertainty on the $\mathrm{N}_{2} \mathrm{H}^{+}(1-0)$ frequency (see text).

Table 2. Ground state spectroscopic constants ${ }^{a}$ of $\mathrm{N}_{2} \mathrm{D}^{+}$.

\begin{tabular}{llccc}
\hline \hline Parameter $(\mathrm{MHz})$ & Laboratory & Radioastronomy & $\mathrm{N}_{2} \mathrm{H}^{+b}$ \\
\hline$v_{\circ}$ & $J=1-0$ & $77109.2697(10)$ & $77109.25811(61)^{c}$ & \\
$e Q q_{1}$ & quadrupole coupling & $-5.6587(42)$ & $-5.6806(31)$ & $-5.6902(21)$ \\
$e Q q_{2}$ & quadrupole coupling & $-1.3713(73)$ & $-1.3461(48)$ & $-1.3586(38)$ \\
$C_{1}$ & spin-rotation & $4.80(84) \times 10^{-3}$ & $9.77(53) \times 10^{-3}$ & $11.8(4) \times 10^{-3}$ \\
$C_{2}$ & spin-rotation & $7.6(11) \times 10^{-3}$ & $6.51(70) \times 10^{-3}$ & $8.7(6) \times 10^{-3}$ \\
\hline
\end{tabular}

${ }^{a}$ Standard errors are reported in parentheses in units of the last quoted digits.

${ }^{b}$ Caselli et al. (1995).

${ }^{c}$ The reported uncertainty is the standard error from the fit, the effective accuracy is $6 \mathrm{kHz}$ (see text).

frequency, due to the errors associated with the velocity determination of the line $\left(0.02 \mathrm{~km} \mathrm{~s}^{-1}\right.$, see Table 2 of Lee et al. 2001). In the same way, the error of the laboratory determination does not account for the possible pressure line shift, which can be estimated in the order of $+10-15 \mathrm{kHz}$ from our preliminary measurements of the effect. The better precision of the hyperfine constants derived from the radiostranomical data is due to their better resolution, which also appears in the higher accuracy of the $C_{1}$ spin-rotation constant.

The analysis was carried out by means of Pickett's SPFIT fitting program (Pickett 1991), which uses the following hyperfine Hamiltonian for plural unequal quadrupole and spinrotation couplings:

$H_{\mathrm{hfs}}=\sum_{i=1}^{N}\left[T^{2}\left(\boldsymbol{q}_{\mathrm{i}}\right) \cdot T^{2}\left(\boldsymbol{Q}_{\mathrm{i}}\right)+T^{1}\left(\boldsymbol{m}_{\mathrm{i}}\right) \cdot T^{1}\left(\boldsymbol{\mu}_{\mathrm{i}}\right)\right]$,

where $T^{k}$ denotes a spherical tensor of rank $k . H_{\mathrm{hfs}}$ accounts for the electrostatic interaction between the quadrupole moment $(\boldsymbol{Q})$ of a nucleus with $I \geq 1$ and the electric field gradient $(\boldsymbol{q})$ at the nucleus, and for the interaction between the magnetic dipole moment $(\boldsymbol{\mu})$ of a nucleus with $I \geq \frac{1}{2}$ and the magnetic field $(\boldsymbol{m})$ due to the molecular rotation. In the present case, the sum should run over all the three nuclei of the molecule. The Deuterium coupling, however, can be neglected, as proved by the following arguments: $i$ ) no more than seven hyperfine components as expected in the case of coupling of both $\mathrm{N}$ nuclei were detected; $i i$ ) the residuals of the fit without $\mathrm{D}$ coupling are well consistent with the measurement uncertainties for both sets of hyperfine data (see Table 1); iii) a prediction of the $\mathrm{D}$ contribution estimated with accurate hyperfine constants of $\mathrm{DC}^{15} \mathrm{~N}$ obtained in a sub-Doppler experiment $\left(e q Q_{\mathrm{D}}=200.6, C_{\mathrm{D}}=-1.9 \mathrm{kHz}\right.$, Cazzoli \& Dore 1990b) gives shifts of the blended hyperfine components of $2 \mathrm{kHz}$ at most: therefore, the hyperfine parameters for outer and inner $\mathrm{N} \mathrm{nu}-$ clei are the only determinable ones. The analysis carried out by Turner (2001) included the Deuterium hyperfine parameters, but this approach led to undetermined values for both $\mathrm{N}$ spin rotation constants and the $\mathrm{D}$ quadrupole constant, and to a large unrealistic value for the $\mathrm{D}$ spin rotation constant.

Table 3 compares the measured higher- $J$ transition frequencies with the old measurements of Sastry et al. (1981) corrected for the Doppler shift. The new data were analyzed with the previously mentioned SPFIT code, which accounts for the shift of the apparent line center from the unperturbed frequency when the hyperfine parameters are fixed to the values derived above from the radio-astronomical data. The newly determined rotational and centrifugal distortion constants agree within $3 \sigma$ with the constants of Sastry et al. (1981), but they are more precise and allow a fairly accurate prediction of the $\mathrm{N}_{2} \mathrm{D}^{+}$transitions occurring in the submillimeter-wave region. In Table 4 are reported the calculated $\Delta F=+1$ hyperfine components of 
Table 3. Rotational transition frequencies and spectroscopic constants of $\mathrm{N}_{2} \mathrm{D}^{+}$.

\begin{tabular}{|c|c|c|c|c|}
\hline$J^{\prime}$ & $J$ & Observed/MHz & Obs.-calc./MHz & New-old/MHz ${ }^{a}$ \\
\hline 1 & 0 & $77109.25811^{b}$ & -0.00031 & \\
\hline 4 & 3 & 308422.267 & 0.016 & 0.057 \\
\hline 5 & 4 & 385516.721 & -0.021 & -0.035 \\
\hline 6 & 5 & 462603.852 & 0.008 & -0.079 \\
\hline \multicolumn{3}{|c|}{ Constant $^{c}$} & This work & Previous $^{d}$ \\
\hline \multicolumn{3}{|c|}{$B / \mathrm{MHz}$ rotational } & $38554.7523(26)$ & $38554.717(14)$ \\
\hline \multicolumn{3}{|c|}{$D / \mathrm{kHz}$ centrifugal distortion } & $61.552(47)$ & $60.81(37)$ \\
\hline
\end{tabular}

${ }^{a}$ Difference between measurements of this work and of Sastry et al. (1981).

${ }^{b}$ Unperturbed (1-0) frequency, its weight (inverse square of the uncertainty) was assumed to be nine times larger than the others in the fit.

${ }^{c}$ Standard errors are reported in parentheses in units of the last quoted digits.

${ }^{d}$ Sastry et al. (1981).

$J+1 \leftarrow J$ transitions, with $J$ in the range $6-11$, whose relative intensity is more than 0.003 ; these components account for more than $97 \%$ of the total line intensity in each transition and are so close in frequency to give rise to a single blended line, whose frequency, computed as a sum of the hyperfine frequencies weighted by their relative intensity, is reported in Table 4 along with its standard error.

For the sake of completeness, we also report the hyperfine frequencies of the two low- $J$ transitions of $\mathrm{N}_{2} \mathrm{D}^{+}$not considered in this work. The $J=2 \leftarrow 1$ and $J=3 \leftarrow 2$ transitions have 40 and 45 separate components, respectively. Table 5 lists the calculated components $(27$ for the $2 \leftarrow 1$, and 25 for the $3 \leftarrow 2$ ), whose relative intensity is more than 0.001 and which together account for more than $99 \%$ of the total line intensity in each transition. Analogous line lists have been reported for $\mathrm{N}_{2} \mathrm{D}^{+}(2-1)$ and (3-2) by Gerin et al. (2001), who used for their computation $\mathrm{N}_{2} \mathrm{H}^{+}$hyperfine parameters instead of the more accurate $\mathrm{N}_{2} \mathrm{D}^{+}$parameters derived and used in the present paper. Finally, Tables 6-8 report the calculated hyperfine frequencies of the three millimeter-wave transitions observed in this work; the $\Delta F=+1$ components blend together in a single line and account for more than $91 \%, 94 \%$, and $96 \%$ of the total line intensity, respectively; $\Delta F=0$ components with relative intensity greater than 0.001 are also reported.

\section{Conclusion}

The analysis of the fully resolved nitrogen hyperfine structure of $\mathrm{N}_{2} \mathrm{D}^{+}$(1-0) observed toward L183, together with laboratory measurements of the same molecular transition, allowed an accurate determination of the hyperfine constants of both outer and inner nitrogen. No contribution of deuterium appears in the $\mathrm{N}_{2} \mathrm{D}^{+}(1-0)$ hyperfine structure. The determined parameters are, in fact, very similar to the $\mathrm{N}_{2} \mathrm{H}^{+}$constants as assumed by Gerin et al. (2001) in their analysis of $\mathrm{N}_{2} \mathrm{D}^{+}(2-1)$ and (3-2). Along with the accurate rotational and centrifugal distortion constants derived from the high frequency laboratory measurements, these constants has been used to predict the hyperfine structure of $\mathrm{N}_{2} \mathrm{D}^{+}$transitions occuring in the submillimeter-wave region.

Acknowledgements. This work was supported by the Italian Ministry of Public Instruction, University and Research and by ASI (contract I/R/044/02)

\section{References}

Aikawa, Y., Ohashi, N., \& Herbst, E. 2003, ApJ, in press

Aikawa, Y., Ohashi, N., Inutsuka, S., Herbst, E., \& Takakuwa, S. 2001, ApJ, 552, 639

Anderson, T. G., Dixon, T. A., Piltch, N. D., et al. 1977, ApJ, 216, L85 Bacmann, A., Lefloch, B., Ceccarelli, C., et al. 2002, A\&A, 389, L6

Bacmann, A., Lefloch, B., Ceccarelli, C., et al. 2003, ApJ, 585, L55

Benson, P. J., Caselli, P., \& Myers, P. C. 1998, ApJ, 506, 743

Bergin, E. A., Alves, J., Huard, T., \& Lada, C. J. 2002, ApJ, 570, L101

Bergin, E. A., Ciardi, D. R., Lada, C. J., et al. 2001, ApJ, 557, 209

Bergin, E. A., \& Langer, W. D. 1997, ApJ, 486, 316

Butner, H. M., Lada, E. A., \& Loren, R. B. 1995, ApJ, 448, 207

Buffa, G., Tarrini, O., Cazzoli, G., \& Dore, L. 1994, Phys. Rev. A, 49, 3557

Caselli, P., Myers, P. C., \& Thaddeus, P. 1995, ApJ, 455, L77

Caselli, P., Walmsley, C. M., Tafalla, M., Dore, L., \& Myers, P. C. 1999, ApJ, 523, L165

Caselli, P., Walmsley, C. M., Terzieva, R., \& Herbst, E. 1998, ApJ, 499, 234

Caselli, P., Walmsley, C. M., Zucconi, A., et al. 2002a, ApJ, 565, 331

Caselli, P., Walmsley, C. M., Zucconi, A., et al. 2002b, ApJ, 565, 344

Cazzoli, G., \& Dore, L. 1990, J. Mol. Spectrosc., 141, 49

Cazzoli, G., \& Dore, L. 1990, J. Mol. Spectrosc., 143, 231

Cazzoli, G., Puzzarini, C., \& Lapinov, A. V. 2003, ApJ, 592, L95

Dalgarno, A., \& Lepp, S. 1984, ApJ, 287, L47

De Lucia, F. C., Herbst, E., Plummer, G. M., \& Blake, G. A. 1982, J. Chem. Phys., 78, 2312

Dore, L., Degli Esposti, C., Mazzavillani, A., \& Cazzoli, G. 1999, Chem. Phys. Lett., 300, 489

Gerin, M., Pearson, J. C., Roueff, E., Falgarone, E., \& Phillips, T. G. 2001, ApJ, 551, L193

Guelin, M., Langer, W. D., \& Wilson, R. W. 1982, A\&A, 107, 107

Jorgensen, J. K., Schöier, F. L., \& van Dishoeck, E. F. 2002, A\&A, 389,908

Kramer, C., Alves, J., Lada, C. J., et al. 1999, A\&A, 342, 257

Lee, J.-E., Evans, N. J., II, Shirley, Y. L., \& Tatematsu, K. 2003, ApJ, 583,789

Lee, C. W., Myers, P. C., \& Tafalla, M. 2001, ApJS, 136, 703

Linsky, J. L., Wood, B. E., Brown, A. Giampapa, M. S., \& Ambruster, C. 1995, ApJ, 455, 670

McKee, C. F. 1989, ApJ, 345, 782

McMullin, J. P., Mundy, L. G., \& Blake, G. A. 1994, ApJ, 437, 305

Millar, T. J., Bennett, A., \& Herbst, E. 1989, ApJ, 340, 906

Pickett, H. M. 1991 J. Mol. Spectrosc., 148, 371

Roberts, H., \& Millar, T. J. 2000, A\&A, 364, 780

Sastry, K. V. L. N., Helminger, P., Herbst, E., \& De Lucia, F. C. 1981, Chem. Phys. Lett., 84, 286

Snyder, L. E., Hollis, J. M., Buhl, D., \& Watson, W. D. 1977, ApJ, 218, L61

Tafalla, M., Myers, P. C., Caselli, P., Walmsley, C. M., \& Comito, C. 2002, ApJ, 569, 815

Turner, B. E. 2001, ApJS, 136, 579

Vrtilek, J. M., Gottlieb, C. A., \& Thaddeus, P. 1987, ApJ, 314, 716

Willacy, K., Langer, W. D., \& Velusamy, T. 1998, ApJ, 507, L171

Williams, J. P., Bergin, E. A., Caselli, P., Myers, P. C., \& Plume, R. 1998, ApJ, 503, 689 